\title{
Effect of Denture-Related Stomatitis Fluconazole Treatment on Oral Candida albicans Susceptibility Profile and Genotypic Variability
}

\author{
Maria Helena Figueiral ${ }^{1, *}$, Patrícia Fonseca ${ }^{1}$, Maria Manuel Lopes ${ }^{2}$, Eugénia Pinto ${ }^{3}$, \\ Teresa Pereira-Leite ${ }^{4}$ and Benedita Sampaio-Maia ${ }^{1,5}$
}

${ }^{I}$ Faculty of Dentistry, Universidade do Porto, Portugal; ${ }^{2}$ Department of Microbiology, Faculty of Pharmacy, Lisbon University, Portugal; ${ }^{3}$ CEQUIMED/CIIMAR, Microbiology Service, Biological Sciences Department, Faculty of Pharmacy, Universidade do Porto, Portugal; ${ }^{4}$ USF-Alcaides, Portugal; ${ }^{5} I N E B / I 3 S$ - Instituto de Engenharia Biomédica, Instituto de Investigação e Inovação em Saúde, Universidade do Porto, Portugal

\begin{abstract}
Denture-related stomatitis (DRS) is the most common condition affecting removable-denture wearers, and Candida albicans the most frequent pathogenic agent. Systemic antifungal treatment is indicated but recurrences are frequent. The aim of this study was to characterize the oral load, fluconazole susceptibility profile and genotypic variability of oral C. albicans isolates from patients with DRS before (T0), immediately after fluconazole treatment (Tat) and after 6-months follow-up (T6m). Eighteen patients presenting DRS and treated with fluconazole were followed at the Faculty of Dentistry of Oporto University. Seventy C. albicans isolates were obtained and identified using standard cultural and biochemical multi-testing. Fluconazole susceptibility was tested by E-test ${ }^{\circledR}$. Microsatellite-primed PCR was performed to assess the genotypic variability of $C$. albicans isolates. The patients' mean age was $58.0 \pm 3.2$ years, and $55.6 \% / 44.4 \%$ had total/partial dentures. Before treatment, $22.2 \%, 44.4 \%$ and $33.3 \%$ of the patients presented DRS type I, II or III, respectively. Fluconazole treatment healed or improved DRS in $77.8 \%$ of the patients, accompanied by an $83.5 \%$ reduction in oral C. albicans load. However, after 6-months, oral C. albicans load increased significantly and DRS severity was similar to the one observed before treatment. Moreover, the prevalence of patients presenting fluconazole resistant isolates of C. albicans increased significantly throughout the study: T0-5.6\%, Tat- $10.0 \%$ and $\mathrm{T} 6 \mathrm{~m}-42.9 \%$. A change in the genotypic variability of $C$. albicans isolates was also verified, being mostly associated to fluconazole susceptibility profile change. In conclusion, fluconazole presents a good short-term DRS treatment efficiency, but may be associated to a long-term emergence of C. albicans fluconazole resistance.
\end{abstract}

Keywords: Candida albicans, denture, denture wearers, denture-related stomatitis, fluconazole resistance, genotypic variability.

\section{INTRODUCTION}

Denture-related stomatitis (DRS) is defined as an inflammatory process of the oral mucosa underlying a removable, partial or total, dental prosthesis. In patients with removable prostheses, the mean prevalence of DRS is $50 \%$ $[1,2]$. Aetiological factors in DRS include the trauma caused by an ill-fitting denture, lack of oral and prosthesis hygiene and a favourable environment for the proliferation of microorganisms [3]. Although bacterial infection, mechanical irritation, and allergic reaction have been proposed as possible causes of DRS, infection with Candida species is often implicated $[1,2,4]$.

Candida spp. are oral commensals present in up to $90 \%$ of healthy persons and Candida albicans (C. albicans) is the most frequent colonizer fungi [5-7]. Previous studies from our group showed that DRS is clearly associated with oral infection by yeasts, mainly $C$. albicans [1], although the same genotype may be found both in DRS patients and in

*Address correspondence to this author at the Rua Dr. Manuel Pereira da Silva, 4200-393 Porto Portugal;

Tel: +351 220901 100; Fax: +351 220901 101;

E-mails:mhsilva@fmd.up.pt and hfigueiral@gmail.com healthy controls [8]. Although DRS is not a particularly severe pathology, it may be a portal of entry for further infections mainly in susceptible patients $[1,2]$.

There is clear evidence that the management of Candidaassociated denture stomatitis is complex due to its multifactorial aetiology [9-11]. Current treatment includes control of denture plaque, and, with patient compliance, some rest periods of denture wearing in addition to the use of antimicrobial agents $[12,13]$. Topical antifungal drugs, such as nystatin, amphotericin B, and miconazole have proved to be effective in DRS improvement [14-16]. However, recurrence rates are high and treatment regimens tend to be extended $[17,18]$. The systemic triazoles, such as fluconazole, are currently the first therapeutic choice for the treatment of this infection $[19,20]$, although progressive recolonization of the palate and the denture fitting surface by yeast, together with a high rate of clinical relapse and recurrence after therapy, have also been reported $[14,21]$. So, the aim of this study was to characterize during a 6-months period the oral load, fluconazole susceptibility profile and genotypic variability of oral $C$. albicans isolates from DRS patients treated with fluconazole therapy. 


\section{MATERIAL AND METHODS}

\section{Study Design and Patient Selection}

Twenty-one patients wearing polymethylmethacrylate maxillary removable prosthesis, with clinical diagnosis of DRS, followed at the outpatient clinic of the Faculty of Dentistry of Oporto University were randomly selected. Patients were assessed clinically and saliva samples were collected for microbiological analysis at the first clinical evaluation and diagnosis (T0), after fluconazole treatment (Tat) and after a 6-month follow-up period (T6m). Fluconazole was prescribed systemically (Fluconazol $50 \mathrm{mg}$, Diflucan ${ }^{\mathbb{B}}$ Pfizer) for 15 days to all patients. After this period, systemic fluconazole was prescribed for further 15 days only to patients who did not improve clinically from DRS.

Ethics Committee of the Faculty of Dentistry of Oporto University reviewed and approved this study. All participants were recruited voluntarily after receiving detailed information on the study protocol. Free and written informed consent was obtained from all participants, according to the Helsinki Declaration.

\section{Clinical Oral Evaluation}

To identify and characterize the different DRS presentations, the Newton classification was used: DRS type I (localized inflammation or hyperaemia points), DRS type II (diffuse erythema) and DRS type III (palate papillary hyperplasia) [22].

Denture hygiene was assessed by modification of the Tarbet index [1, 4, 23]. The patients were classified according to the total denture surface covered with microbial plaque evaluated by a dental plaque disclosing solution (Dento-plaque ${ }^{\circledR}$, Pierre-Fabre Dermo-Cosmetique) in the following groups: poor denture hygiene, $>76 \%$; insufficient denture hygiene, 26 to $75 \%$, and good denture hygiene, $<25 \%$.

Additionally, before the oral clinical evaluation, unstimulated whole saliva was collected in a sterile container at least $2 \mathrm{~h}$ after eating, tooth brushing, mouth washing or smoking.

\section{Candida albicans Isolation and Quantification}

Saliva samples from all patients were serially diluted with $0.9 \%$ sterile $\mathrm{NaCl}$ solution until $10^{-1}$ and immediately plated in triplicate in a selective and differential culture medium, CHROMagar CandidaTM ${ }^{\mathbb{B}}$ (Becton Dickinson, Sparks, USA). Afterwards, plates were incubated aerobically for $48 \mathrm{~h}$ at $37^{\circ} \mathrm{C}$. The total number of green-pigmented colonies (Candida albicans) were counted and isolated. The lower limit of detection was $1 \mathrm{CFU} / \mathrm{mL}$. C. albicans isolates were identified based on germ-tube formation, chlamydospore production and carbohydrate assimilation using the ID 32C system (BioMerieux, Marcy l'Etoile, France). The yeasts identified as C. albicans were also screened for their ability to grow on Sabouraud dextrose agar at $45^{\circ} \mathrm{C}$ for $48 \mathrm{~h}$ and xylose assimilation, to distinguish from $C$. dubliniensis [24]. Several colonies of each different phenotype were selected and kept frozen at $-70^{\circ} \mathrm{C}$, in sterile water with $30 \%$ glycerol. Prior to testing, the yeasts were subcultured in
Sabouraud dextrose agar (BioMerieux, Marcy l'Etoile, France) and incubated at $37^{\circ} \mathrm{C}$ for two days. A total of $70 \mathrm{C}$. albicans isolates were obtained at T0, Tat and T6m from all patients. C. dubliniensis FFUL 21 and Candida albicans ATCC 90028 were used as internal controls for the identification tests.

\section{Antifungal Susceptibility Testing}

Susceptibility testing to fluconazole was performed by Etest $^{\circledR}$ (BioMerieux, Marcy l'Etoile, France), according to the manufacturer's instructions. C. albicans isolates were classified accordingly to the proposed in vitro breakpoints to fluconazole: susceptible, minimal inhibitory concentration (MIC) $\leq 8 \mathrm{mg} / \mathrm{L}$; susceptible-dose dependent (S-DD), MIC between 16 to $32 \mathrm{mg} / \mathrm{L}$; and resistant, $\mathrm{MIC} \geq 64 \mathrm{mg} / \mathrm{L}$.

\section{Microsatellite-Primed PCR}

For microsatellite-primed PCR genomic DNA was extracted in accordance with Treco [25]. Nucleic acid purity and concentration were assessed by spectroscopy at 260 and $280 \mathrm{~nm}$. A single repeat sequence of the phage M13, 5'-GAG GGT GGC GGT TCT-3' was used in the microsatelliteprimed PCR experiments, because the array of DNA fragments presented high intensity bands and identical patterns of DNA fragments were detected with replicate samples, showing to be the best primer tested [26]. Synthetic oligodeoxyribonucleotide molecules were prepared by OligoExpress (Paris, France). Amplification reactions were performed in volumes of $50 \mathrm{~mL}$ containing $25 \mathrm{ng}$ template DNA, $10 \mathrm{mmol} / \mathrm{L}$ Tris- $\mathrm{HCl}(\mathrm{pH} 8.3), 50 \mathrm{mmol} / \mathrm{L} \mathrm{KCl}, 4 \mathrm{mmol} / \mathrm{L}$ $\mathrm{MgCl}_{2}, 200 \mathrm{mmol} / \mathrm{L}$ of each dNTPs, $1 \mathrm{mmol} / \mathrm{L}$ of primer, and 2.5U Taq DNA polymerase. Except for dNTPs, obtained from Amersham Pharmacia Biotech (Uppsala, Sweden), all PCR reagents were purchased from Perkin Elmer Corp., Applied Biosystems (New Jersey, USA). The samples were amplified in a Biometra T1 termocycler (Göttingen, Germany), and the thermal cycling parameters were an initial denaturation at $95^{\circ} \mathrm{C}$ for $3 \mathrm{~min}$, followed by 35 cycles of denaturation at $95^{\circ} \mathrm{C}$ for $45 \mathrm{~s}$, annealing at $46^{\circ} \mathrm{C}$ for $40 \mathrm{~s}$, and extension at $72^{\circ} \mathrm{C}$ for $30 \mathrm{~s}$ with final extension at $72^{\circ} \mathrm{C}$ for 5 minutes. PCR products were analysed by electrophoresis with agarose gel stained with ethidium bromide. DNA Ladder and pBR322 DNA-BstNI digest (New England Biolabs, Beverly, Mass.) were used as molecular size standards. To ensure the reproducibility of the results, reference strains were systematically run as controls together with negative controls (no DNA). The profiles for each isolate were compared visually with identical band patterns deemed to represent the same genotype.

\section{Statistical Analysis}

The statistical analysis was performed using $\mathrm{IBM}^{\circledR}$ SPSS $^{\circledR}$ version 19.0 (Statistical Package for Social Sciences). The categorical variables were described through absolute and relative frequencies (\%) and analysed by Chi-square independence test. Continuous variables were described using mean \pm standard error (SEM) and analysed by Anova followed by students' t-test. Statistical significance was assumed when the $\mathrm{p}$ values were less than 0.05 . 


\section{RESULTS}

The first clinical evaluation and diagnosis (T0) was performed to 21 patients presenting a mean age of 57.6 years with a standard error of 3.1 years; $28.6 \%$ were males and $71.4 \%$ were females. Three patients $(14.3 \%)$ did not conclude the study due to different reasons - death, hospitalization and unknown whereabouts - and were excluded thus from the analysis. The characterization of the group of participants followed during the 6-month period is shown in Table $1(n=18)$. All patients had dentures of polymethylmethacrylate resin. At the time of the first clinical evaluation most of the patients presented DRS type II and insufficient denture hygiene (Table 1).

Table 1. Characterization of patients with denture-related stomatitis (DRS) regarding age, denture type and denture hygiene at the time of first diagnosis (TO).

\begin{tabular}{|c|c|}
\hline & $\begin{array}{c}\text { DRS patients } \\
(\mathbf{n}=\mathbf{1 8})\end{array}$ \\
\hline \hline Age, years & $58.0 \pm 3.2$ \\
\hline Gender, male / female & $33.3 \% / 66.6 \%$ \\
\hline Total / partial denture & $55.6 \% / 44.4 \%$ \\
\hline DRS type I / II / III & $22.2 \% / 44.4 \% / 33.3 \%$ \\
\hline Poor / insufficient / good denture hygiene & $16.7 \% / 66.7 \% / 16.7 \%$ \\
\hline
\end{tabular}

Values are mean \pm sem or prevalence $(\%)$.

After 15 days of fluconazole treatment, $55.6 \%$ of the patients healed or improved DRS (Table 2). The remaining $44.4 \%$ presented DRS type I or II, evenly. These patients were prescribed further 15 days of fluconazole treatment. Half of the patients with 30 days of fluconazole treatment healed or improved DRS. In total, fluconazole therapy healed or improved DRS in $77.8 \%$ of the patients, regardless of the duration of the treatment. After the fluconazole treatment, $38.9 \%$ of the patients presented no clinical evidence of DRS, $44.4 \%$ presented DRS type I, $16.7 \%$ presented DRS type II, and no patient presented DRS type III (Fig. 1). After the 6-months follow up period, the DRS worsened in most of the evaluated patients and DRS severity was similar to that observed before treatment (Fig. 1). The prevalence of DRS type changed significantly throughout the study (Fig. 1, $\mathrm{p}=0.001)$.

Table 2. Fluconazole response of the followed up patients.

\begin{tabular}{|c|c|c|c|}
\hline $\begin{array}{c}\text { Fluconazole } \\
\text { treatment period }\end{array}$ & Healed & Improved & $\begin{array}{c}\text { Not } \\
\text { improved }\end{array}$ \\
\hline \hline 15 days & $27.8 \%$ & $27.8 \%$ & $44.4 \% *$ \\
\hline Plus 15 days & $11.1 \%$ & $11.1 \%$ & $22.2 \%$ \\
\hline
\end{tabular}

*These patients did further 15 days of fluconazole therapy.

At T0, the oral C. albicans prevalence in the evaluated patients was $94.4 \%$ and the mean load was $139.9 \pm 27.9 \mathrm{CFU} / \mathrm{mL}$ of saliva. Fluconazole treatment re- duced oral $C$. albicans prevalence and load in saliva, but at T6m the C. albicans prevalence increased, as did the C. albicans load (Fig. 2). The oral C. albicans prevalence profile differed significantly throughout the study $(\mathrm{p}=0.015)$.

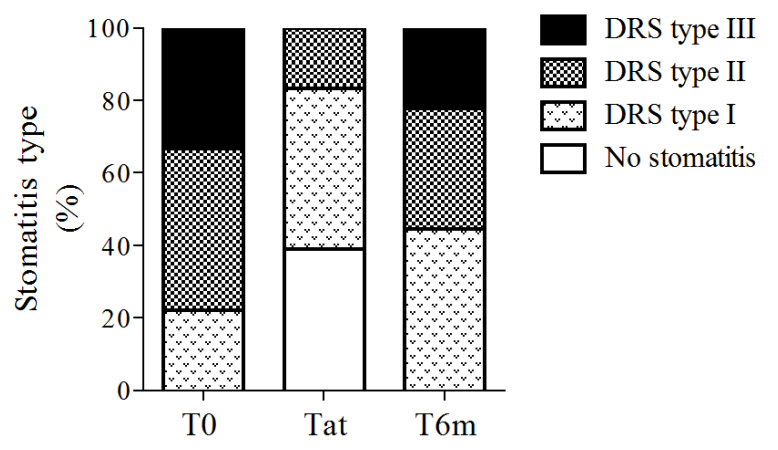

Fig. (1). Denture-related stomatitis (DRS) type at the time of stomatitis diagnosis (T0), after treatment (Tat) and 6-months after diagnosis (T6m). Bars represent prevalence.

At T0, the fluconazole susceptible dose-dependent and resistant isolates of $C$. albicans represented $5.6 \%$ each, whereas the susceptible isolates represented $88.9 \%$ (Fig. 3). After the 6-month follow-up period the C. albicans resistant isolates represented $42.9 \%$, whereas the susceptible isolates represented $57.1 \%$ (Fig. 3).

The analysis of genotypic variability of $C$. albicans isolates, using primer M13, generated profiles with 6 to 14 bands varying between 400 and $1,800 \mathrm{bp}$. From all evaluated patients that presented $C$. albicans counts in more than in one evaluation point, $42.9 \%$ showed a change in genotypic variability of $C$. albicans isolates throughout the study. Two examples of $C$. albicans profile change from T0 to T6m are shown in Fig. (4). Patient 1 presents two different genetic profiles, whereas patient 2 presents 3 different $C$. albicans genetic profiles. Identical profiles were obtained for all replicate samples, confirming the high reproducibility of this method. From this group of patients presenting C. albicans genotypic variability of M13 primer throughout the study, $83.3 \%$ had at least one C. albicans isolate resistant to fluconazole.

\section{DISCUSSION}

The present work intended to evaluate the effect of fluconazole treatment on short-term and long-term clinical development of DRS and C. albicans oral colonization profile.

Several factors may contribute to the onset and worsening of DRS, namely poor denture hygiene, continuous wearing of removable dentures, accumulation of denture plaque, bacterial and yeast contamination of denture surface and mucosal trauma due to poor-fitting dentures. In the present study each patient was his own control, since patients were evaluated before and after fluconazole treatment. Changes were not observed throughout the study in denture substitution or adjustment, denture hygiene promotion and denture daily wearing period, factors that could influence DRS progression besides fluconazole therapy.

There is a large body of evidence indicating that Candida is able to adhere to acrylic resin dentures, namely to 


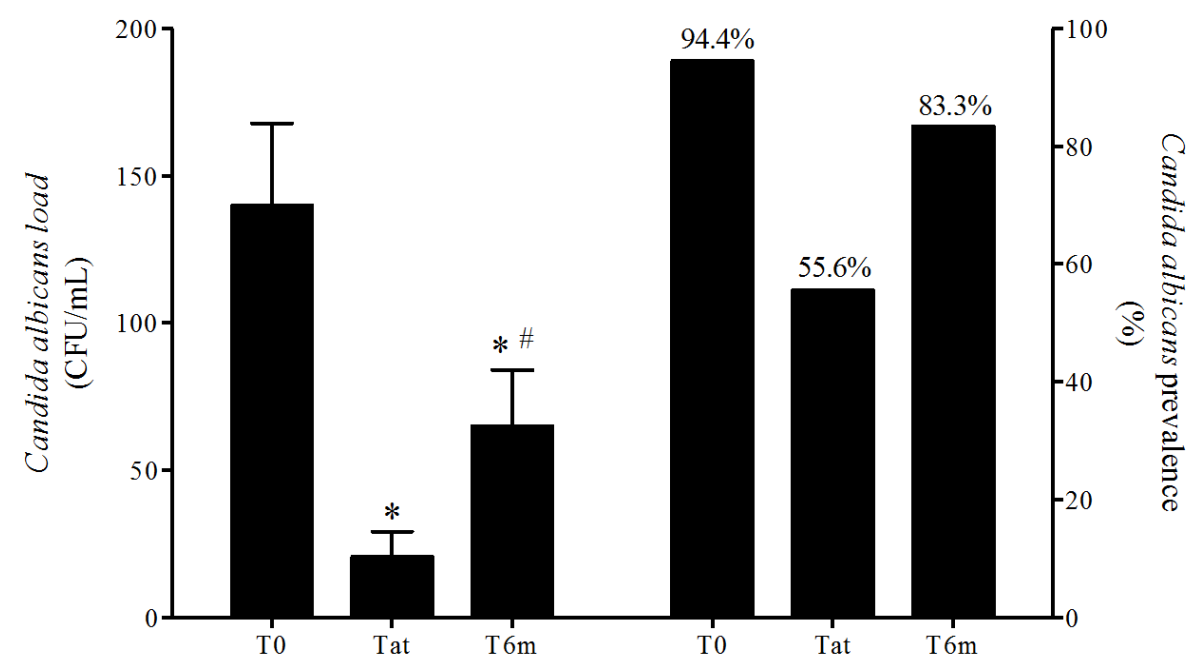

Fig. (2). Oral Candida albicans load and prevalence at the time of stomatitis diagnosis (T0), after treatment (Tat) and 6-months after diagnosis (T6m). Left bars represent means and error bars represent SD. $p$ values were calculated using Anova ( $\mathrm{p}=0.00038)$ followed by students' ttest. ${ }^{*} \mathrm{p}<0.05$, significantly different from $\mathrm{T} 0 ;{ }^{\#} \mathrm{p}<0.05$, significantly different from Tat. Right bars represent prevalence.

\section{Candida albicans}

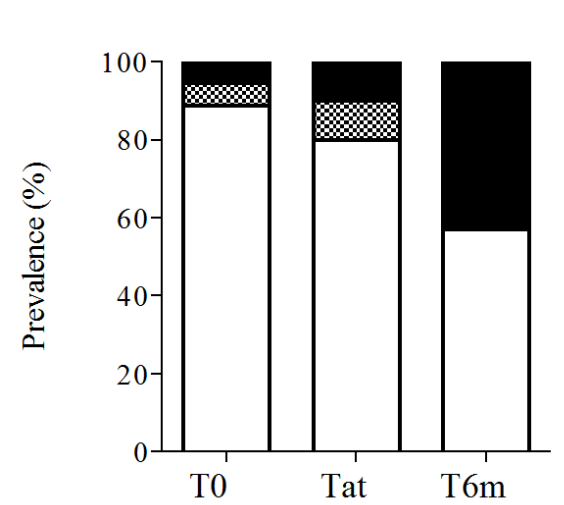

Fluconazole resistant

\% Fluconazole susceptible dose-dependent

$\square$ Fluconozole susceptible

Fig. (3). Prevalence of fluconazole susceptibility profile of Candida albicans isolates at the time of stomatitis diagnosis (T0), after treatment (Tat) and 6-months after diagnosis (T6m). Bars represent prevalence.

polymethylmethacrylate - PMMA, forming a biofilm $[27$, 28]. As expected and in agreement with previous reports, denture wearer patients presented high rates of $C$. albicans oral colonization $(94.4 \%)$ at the time of the first clinical evaluation (T0) [29-31]. This denture biofilm provides a source of microorganisms that is continuously exposed to the oral mucosa. This is the first step that may lead to the development of the infectious process and that may ultimately result in varying degrees of denture stomatitis of the adjacent mucosa $[29,32]$.

As expected, fluconazole treatment significantly reduced the oral $C$. albicans colonization and greatly improved the severity of DRS in the studied population [29, 33, 34]. However, these effects were observed only immediately after treatment (Tat). After the 6-month follow-up period (T6m), either oral $C$. albicans colonization or DRS severity profiles were similar to the ones observed before fluconazole treatment, revealing a good short-term but not a good long-term fluconazole DRS treatment efficiency. This fact probably reflects recontamination by residual yeasts that are present on the denture surfaces [29].

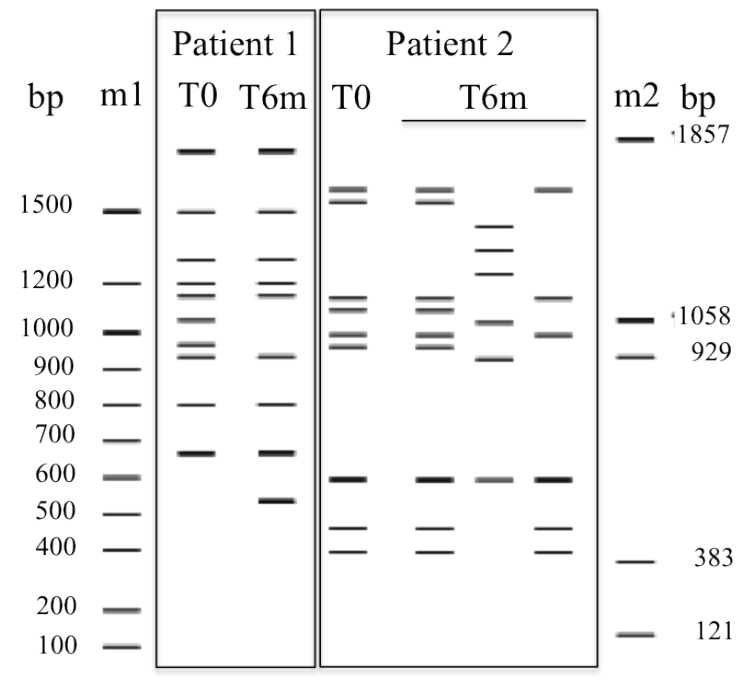

Fig. (4). Genotypic variability of Candida albicans isolates from two different patients studied by MSP-PCR with the primer M13 at the time of DRS diagnosis (T0), and 6-months after diagnosis (T6m). Molecular size markers used were DNA Ladder (m1) and pBR322 DNA-BstNI digest (m2). 
Additionally, the C. albicans oral population appear to change over time due to the increase of $C$. albicans isolates resistant to fluconazole 6-months after therapy. This hypothesis was reinforced by the high rate of genetic variability found in $C$. albicans isolates within each patient when isolates collected before and 6-months after fluconazole treatment were compared. Notwithstanding, no specific C. albicans resistant genes were analysed, such as the efflux pumps [35]. Increasing scientific literature addresses this phenomenon of antimicrobial resistance induced by therapeutic treatment regarding non-oral infections [36]. In oral cavity, antimicrobial resistance is still scarce; however, dental medical doctors should be aware of its increasing importance and future implication. Despite the good short-term fluconazole efficiency in the treatment of DRS, the fluconazolegeneralized prescription should be avoided to reduce the emergence of resistant strains of $C$. albicans and the increasing episodes of DRS recurrences. Therefore, other therapeutic or prevention strategies should be implemented before fluconazole is recommended, namely denture disinfection together with denture hygiene education and surveillance, denture adjustment or replacement [20]. In addition, new developments related to denture materials are focusing on means to reduce development of adherent biofilms. These developments may be valuable in reducing yeast colonization, and could lead to reductions in denture stomatitis with appropriate denture hygiene [29, 37].

Nevertheless, some clinical scenarios may require fluconazole use, namely in DRS in immunosuppressed patients at a risk of a systemic infection or patients with persistent severe symptomatologic DRS [20].

\section{CONCLUSION}

In conclusion, fluconazole presents a good short-term, but not a good long-term, DRS treatment efficiency. Also fluconazole treatment of DRS may be associated to a longterm emergence of oral C. albicans fluconazole resistance. Other therapeutic or prevention strategies should be implemented before fluconazole is prescribed, namely denture disinfection, together with denture hygiene education and surveillance, as well as denture adjustment or replacement.

\section{CONFLICT OF INTEREST}

The authors confirm that this article content has no conflict of interest.

\section{ACKNOWLEDGEMENTS}

Declared none.

\section{REFERENCES}

[1] Figueiral MH, Azul A, Pinto E, Fonseca PA, Branco FM, Scully C. Denture-related stomatitis: Identification of aetiological and predisposing factors - a large cohort. J Oral Rehabil 2007; 34: 44855.

[2] Pinto E, Ribeiro IC, Ferreira NJ, Fortes CE, Fonseca PA, Figueiral MH. Correlation between enzyme production, germ tube formation and susceptibility to fluconazole in Candida species isolated from patients with denture-related stomatitis and control individuals. J Oral Pathol Med 2008; 37: 587-92.
[3] Zissis A, Yannikakis S, Harrison A. Comparison of denture stomatitis prevalence in 2 population groups. Int $\mathrm{J}$ Prosthodont 2006; 19: 621-5.

[4] Figueiral MH. Estomatite Protética: Identificação e caracterização dos factores etiológicos e predisponentes. PhD dissertation. Faculty of Dental Medicine: University of Porto 2000 [in Portuguese].

[5] Ghannoum MA, Jurevic RJ, Mukherjee PK, et al. Characterization of the oral fungal microbiome (mycobiome) in healthy individuals. PLoS Pathog 2010; 6: e1000713.

[6] Monteiro-da-Silva F, Araujo R, Sampaio-Maia B. Interindividual variability and intraindividual stability of oral fungal microbiota over time. Med Mycol 2014; 52: 498-505.

[7] Monteiro-da-Silva F, Sampaio-Maia B, Pereira Mde L, Araujo R. Characterization of the oral fungal microbiota in smokers and nonsmokers. Eur J Oral Sci 2013; 121: 132-5.

[8] Costa F, Manaia CM, Figueiral MH, Pinto E. Genotypic analysis of Candida albicans isolates obtained from removable prosthesis wearers. Lett Appl Microbiol 2008; 46: 445-9.

[9] Darwazeh AM, Al-Refai S, Al-Mojaiwel S. Isolation of Candida species from the oral cavity and fingertips of complete denture wearers. J Prosthet Dent 2001; 86: 420-3.

[10] Sampaio-Maia B, Figueiral MH, Sousa-Rodrigues P, Fernandes $\mathrm{MH}$, Scully C. The effect of denture adhesives on Candida albicans growth in vitro. Gerodontology 2012; 29: e348-56.

[11] Webb BC, Thomas CJ, Willcox MD, Harty DW, Knox KW Candida-associated denture stomatitis. Aetiology and management: A review. Part 3. Treatment of oral candidosis. Aust Dent J 1998; 43: 244-9.

[12] Iacopino AM, Wathen WF. Oral candidal infection and denture stomatitis: A comprehensive review. J Am Dent Assoc 1992; 123: 46-51.

[13] Shay K, Truhlar MR, Renner RP. Oropharyngeal candidosis in the older patient. J Am Geriatr Soc 1997; 45: 863-70.

[14] Bissell V, Felix DH, Wray D. Comparative trial of fluconazole and amphotericin in the treatment of denture stomatitis. Oral Surg Oral Med Oral Pathol 1993; 76: 35-9.

[15] Dorocka-Bobkowska B, Konopka K. Susceptibility of candida isolates from denture-related stomatitis to antifungal agents in vitro. Int J Prosthodont 2007; 20: 504-6.

[16] Martinez-Beneyto Y, Lopez-Jornet P, Velandrino-Nicolas A, Jornet-Garcia V. Use of antifungal agents for oral candidiasis: Results of a national survey. Int J Dent Hyg 2010; 8: 47-52.

[17] Cross LJ, Williams DW, Sweeney CP, Jackson MS, Lewis MA, Bagg J. Evaluation of the recurrence of denture stomatitis and Candida colonization in a small group of patients who received itraconazole. Oral Surg Oral Med Oral Pathol Oral Radiol Endod 2004; 97: 351-8.

[18] Ramage G, Tomsett K, Wickes BL, Lopez-Ribot JL, Redding SW. Denture stomatitis: A role for Candida biofilms. Oral Surg Oral Med Oral Pathol Oral Radiol Endod 2004; 98: 53-9.

[19] Martin-Mazuelos E, Aller AI, Romero MJ, et al. Response to Fluconazole and itraconazole of Candida spp. in denture stomatitis. Mycoses 1997; 40: 283-9.

[20] Pappas PG, Kauffman CA, Andes D, et al. Clinical practice guidelines for the management of candidiasis: 2009 update by the Infectious Diseases Society of America. Clin Infect Dis 2009; 48: 503-35.

[21] Karyotakis NC, Anaissie EL. The new antifungal azoles: Fluconazole and itraconazole. Curr Opin Infect Dis 1994; 7: 65866.

[22] Budtz-Jorgensen E, Bertram U. Denture stomatitis. I. The etiology in relation to trauma and infection. Acta Odontol Scand 1970; 28: 71-92.

[23] Tarbet WJ. Denture plaque: Quiet destroyer. J Prosthet Dent 1982; 48: 647-52.

[24] Gales AC, Pfaller MA, Houston AK, et al. Identification of Candida dubliniensis based on temperature and utilization of xylose and alpha-methyl-D-glucoside as determined with the API 20C AUX and vitek YBC systems. J Clin Microbiol 1999; 37: 3804-8.

[25] Treco DA. Preparation of yeast DNA, RNA and proteins. In: Ausebel FM, Brent R, Kingston RE, et al., Eds. Current Protocols in Molecular Biology. USA: Wiley and Sons 1989.

[26] Bartie KL, Williams DW, Wilson MJ, Potts AJ, Lewis MA. PCR fingerprinting of Candida albicans associated with chronic 
hyperplastic candidosis and other oral conditions. J Clin Microbiol 2001; 39: 4066-75.

[27] Chandra J, Mukherjee PK, Leidich SD, et al. Antifungal resistance of candidal biofilms formed on denture acrylic in vitro. J Dent Res 2001; 80: 903-8

[28] Pereira-Cenci T, Del Bel Cury AA, Crielaard W, Ten Cate JM. Development of Candida-associated denture stomatitis: New insights. J Appl Oral Sci 2008; 16: 86-94.

[29] Gendreau L, Loewy ZG. Epidemiology and etiology of denture stomatitis. J Prosthodont 2011; 20: 251-60.

[30] Radford DR, Challacombe SJ, Walter JD. Denture plaque and adherence of Candida albicans to denture-base materials in vivo and in vitro. Crit Rev Oral Biol Med 1999; 10: 99-116.

[31] Vanden Abbeele A, de Meel H, Ahariz M, Perraudin JP, Beyer I, Courtois P. Denture contamination by yeasts in the elderly. Gerodontology 2008; 25: 222-8.
[32] Caldas IM, Grams AC, Afonso A, Magalhaes T. Oral injuries in victims involving intimate partner violence. Forensic Sci Int 2012; 221: $102-5$.

[33] Kabir MA, Ahmad Z. Candida infections and their prevention. ISRN Preventive Med 2013; 2013: 763628.

[34] Lombardi T, Budtz-Jorgensen E. Treatment of denture-induced stomatitis: A review. Eur J Prosthodont Restor Dent 1993; 2: 1722.

[35] Morschhauser J. Regulation of multidrug resistance in pathogenic fungi. Fungal Genet Biol 2010; 47: 94-106.

[36] Shorr AF, Chung K, Jackson WL, Waterman PE, Kollef MH. Fluconazole prophylaxis in critically ill surgical patients: A metaanalysis. Crit Care Med 2005; 33: 1928-35.

[37] von Fraunhofer JA, Loewy ZG. Factors involved in microbial colonization of oral prostheses. Gen Dent 2009; 57: 136-43.

Received: November 08, 2014

Revised: December 15,2014

Accepted: December 18, 2014

(C) Figueiral et al.; Licensee Bentham Open.

This is an open access article licensed under the terms of the Creative Commons Attribution Non-Commercial License (http://creativecommons.org/licenses/by-nc/3.0/) which permits unrestricted, non-commercial use, distribution and reproduction in any medium, provided the work is properly cited. 